\title{
Differences in the prevalence of prediabetes, undiagnosed diabetes and diagnosed diabetes and associated factors in cohorts of Brazilian and English older adults
}

\author{
Eilane Souza Marques dos Santos ${ }^{1}$, Roberta de Oliveira Máximo², \\ Fabíola Bof de Andrade ${ }^{3} \odot$, Cesar de Oliveira ${ }^{4}$, Maria Fernanda Lima-Costa ${ }^{3}$ and \\ Tiago da Silva Alexandre ${ }^{1,2,5, *}$ (i) \\ 'Gerontology Department, Federal University of Sao Carlos, Rodovia Washington Luís, km 235, SP-310, 13565-905 \\ Sao Carlos, Sao Paulo, Brazil: ${ }^{2}$ Postgraduate Programme in Physical Therapy, Federal University of Sao Carlos, \\ Sao Carlos, Sao Paulo, Brazil: ${ }^{3}$ René Rachou Research Institute, Oswaldo Cruz Foundation, Belo Horizonte, Minas \\ Gerais, Brazil: ${ }^{4}$ Department of Epidemiology and Public Health, University College London, London, UK: \\ ${ }^{5}$ Postgraduate Programme in Gerontology, Federal University of Sao Carlos, Sao Carlos, Sao Paulo, Brazil
}

Submitted 31 March 2020: Final revision received 27 July 2020: Accepted 11 August 2020

\begin{abstract}
Objective: To analyse differences in the prevalence of prediabetes (PD), undiagnosed diabetes (UDD) and diagnosed diabetes (DD) and associated factors between Brazilian and English older adults.

Design: Cross-sectional study.

Setting: England and Brazil.

Participants: 5301 participants of the English Longitudinal Study of Ageing study and 1947 participants of the Brazilian Longitudinal Study of Aging study classified as non-diabetics, PD, UDD and DD.

Results: The prevalence of PD, UDD and DD was 48.6, 3 and 9.6\% in England and 33, 6 and $20 \%$ in Brazil. In England, the increase in age, non-white skin colour, smoking, general obesity and abdominal obesity were associated with PD, UDD and DD, whereas hypertriglyceridaemia, low HDL levels, hypertension and stroke were associated with UDD and DD. In Brazil, the increase in age was associated with DD and UDD, non-white skin colour and smoking were associated with UDD and abdominal obesity and hypertriglyceridaemia were associated with all three conditions. CVD in England and schooling in Brazil were associated with PD and DD. A sedentary lifestyle was associated with DD in both samples.

Conclusions: The prevalence of diabetes was higher in the Brazilian sample. Different associated factors were found in the two samples, which may be related to differences in nutritional transition, access to healthcare services and the use of such services.
\end{abstract}

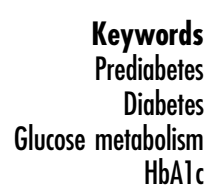

increases the risk of complications ${ }^{(3)}$. The diagnosis of the disease in individuals older than 50 years of age is often hindered due to the masking of the symptoms resulting from age-related changes ${ }^{(4,5)}$. Moreover, individuals with prediabetes (PD) have a 20-fold greater risk of developing diabetes mellitus $^{(1,6)}$. Although PD has outcomes of the typical microvascular complications found in diabetes mellitus (nephropathy, retinopathy and neuropathy), this condition has few obvious clinical signs ${ }^{(7,8)}$ and can last for more than a decade. 
The prevalence of PD, UDD and DD can differ substantially between countries depending on socio-demographic characteristics. Low schooling, low income, a sedentary lifestyle and an inadequate $\operatorname{diet}^{(9)}$, together with the difficulty in identifying individuals with diabetes mellitus, are aggravating factors in developing countries ${ }^{(10)}$. To the best of our knowledge, however, no studies have made comparisons of these aspects between developed and developing countries beyond the determination of different prevalence rates ${ }^{(10-14)}$.

We hypothesise that low-middle-income countries may have a higher prevalence of PD, UDD and DD due to adverse health and socio-demographic factors, as well as rapid and intense nutritional transition accompanied by less effective health policies and healthcare system.

Such comparisons are important and can assist in identifying differences among factors associated with diabetes as well as opportunities to minimise such differences. Therefore, the aim of the present study was to analyse differences in the prevalence of PD, UDD and DD and associated factors between English and Brazilian individuals 50 years of age or older.

\section{Methods}

\section{Data sources: ELSA and ELSI}

Data came from the English Longitudinal Study of Ageing (ELSA) and the Brazilian Longitudinal Study of Aging (ELSI (Estudo Longitudinal de Saúde e Bem-Estar dos Idosos Brasileiros)). They are international harmonised (sister) ageing cohorts that not only provide data for individual countries but also offer the valuable opportunity for

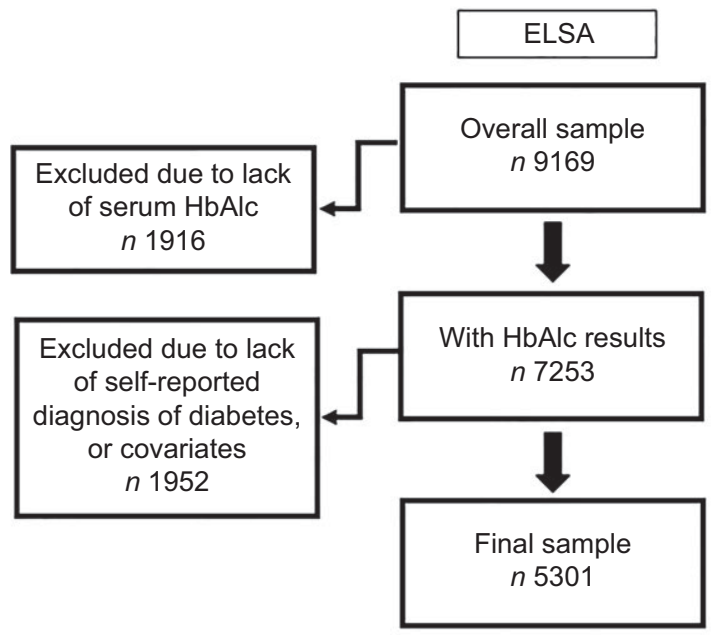

Fig. 1 Sample selection flowchart at ELSA and ELSI studies cross-national comparisons. ELSA is an ongoing panel study commenced in 2002 involving communitydwelling individuals in England aged 50 years or older. ELSA has a nationally representative sample using a multi-stage stratified probability sampling design ${ }^{(15,16)}$. ELSI is a home-based longitudinal study conducted with a representative sample of individuals aged 50 years or older from 70 municipalities across different geographical regions of Brazil. Initiated in 2015-2016, a comprehensive sampling procedure involved different stages of selection, that is, municipalities, census sectors and households. An inverse sampling process was adopted, with 9412 participants.

For the present analysis, we selected participants from the sixth wave of ELSA (conducted in 2012-2013) and the baseline of ELSI. The eligibility criteria for the current analysis were the determination of serum glycated hemoglobin (HbA1c), information on a medical diagnosis of diabetes mellitus and data on the variables of interest. All participants of ELSA were eligible for laboratory exams. However, 1916 out of the 9169 eligible individuals did not have an HbA1c measurement. A probabilistic subsample of 4000 ELSI participants was selected to have blood samples collected, with HbA1c results in 59\% (2360) of these individuals. Further information on the sampling process of these two studies can be found in previous publications ${ }^{(16,17)}$.

We have further excluded 1952 individuals from ELSA and 413 individuals from ELSI for the lack of information on self-reported doctor-diagnosed diabetes or covariates. The final sample in the present study was composed of 5301 ELSA participants and 1947 ELSI participants. Figure 1 shows the sample selection flowchart.

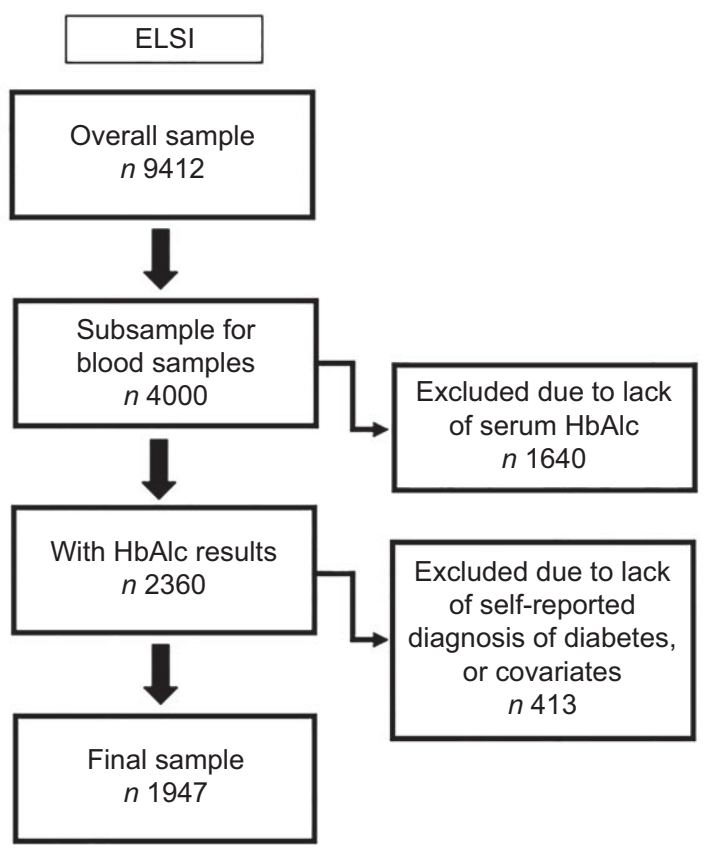


The current study followed the Strengthening the Reporting of Observational Studies in Epidemiology reporting guideline ${ }^{(18)}$.

\section{Dependent variable}

The dependent variable was diabetes mellitus categorised according to self-report and the criteria of the American Diabetes Association for serum HbA1c. The participants were classified as non-diabetic (no self-report of diabetes and $\mathrm{HbA} 1 \mathrm{c}<5.7 \%$ ), prediabetic (no self-report of diabetes and HbA1c $\geq 5.7$ and $<6.5 \%$ ), undiagnosed diabetic (no self-report of diabetes and $\mathrm{HbA} 1 \mathrm{c} \geq 6.5 \%)$ and diagnosed diabetic (self-reported diabetes independently of HbA1c) ${ }^{(8)}$. Diagnosis tests for serum HbA1c were performed in laboratories using the method certified by the National Glycohemoglobin Standardization Program and standardised to the Diabetes Control and Complications Trial reference assay. The present study adheres to the $2012^{(19)}$ and $2015^{(20)}$ editions of the American Diabetes Association for the ELSA and ELSI studies, respectively. Both editions followed the same criteria to diagnose diabetes.

\section{Variables of interest}

The variables of interest were those previously defined as being associated with diabetes mellitus, including sociodemographic characteristics, health-related behaviours, anthropometrics, self-reported health conditions and serum cholesterol $^{(21)}$.

Socio-demographic characteristics were age (in years), sex, schooling $(0-11,12-13$ or 14 years or more), marital status (married $v$. single, divorced or widowed), selfdeclared skin colour (white/non-white) and family income (quintiles).

Heath-related behaviours as alcohol intake (nondrinker, frequent drinker (two to six times per week) or daily drinker) and smoking status (non-smoker, ex-smoker or current smoker) were assessed similarly in both studies. Physically inactive individuals were considered those who performed $<150 \mathrm{~min}$ of physical activity per week measured using the Brazilian version of the International Physical Activity Questionnaire in ELSI. In ELSA, physically inactive individuals were those who did not practice any physical activity at least once a week according to the modified version of the Physical Activity and Sedentary Behaviour Assessment Questionnaire ${ }^{(22)}$.

Anthropometrics measurements collected were body mass index (BMI) and waist circumference. The BMI was calculated by dividing weight in kilograms by height in meters squared $\left(\mathrm{kg} / \mathrm{m}^{2}\right)$ and classified as follows: underweight $\left(<18.5 \mathrm{~kg} / \mathrm{m}^{2}\right)$, ideal range $\left(18.5\right.$ to $\left.<25 \mathrm{~kg} / \mathrm{m}^{2}\right)$, overweight $\left(25\right.$ to $\left.<30 \mathrm{~kg} / \mathrm{m}^{2}\right)$ or obese $\left(\geq 30 \mathrm{~kg} / \mathrm{m}^{2}\right)$. Waist circumference was measured using a metric tape at the midpoint between the lowest rib and the upper edge of the iliac crest at the end of the expiratory phase ${ }^{(23)}$. Abdominal obesity was defined based on waist circumference: $>102 \mathrm{~cm}$ for men and $>88 \mathrm{~cm}$ for women ${ }^{(24)}$.

Clinical conditions based on self-reported doctor diagnosis of hypertension, CVD and stroke were obtained. The biochemical measures included were serum high density lipoprotein (HDL) $(<1.03 \mathrm{mmol} / \mathrm{l}$ for men and $<1.29 \mathrm{mmol} / \mathrm{l}$ for women) and triacylglycerol (TAG) $(\geq 1.69 \mathrm{mmol} / \mathrm{l})^{(25)}$.

\section{Statistical analysis}

The prevalence was calculated with $95 \%$ CI. We performed the direct standardization method having the ELSA sample as the standard population to calculate the standardised prevalence for the ELSI study, adjusting it by age, sex and schooling years ${ }^{(26)}$. Differences in the characteristics of the sample according to diabetes status (non-diabetic, prediabetic, undiagnosed diabetic and diagnosed diabetic) in ELSA were evaluated using the $\chi^{2}$ test, ANOVA and Tukey's post-hoc test. These differences in ELSI were evaluated using the $\chi^{2}$ test with the Rao-Scott correction and the Rao and Scott Wald test, as ELSI has a weighted sample. The multinomial regression model was used to allow the analysis of factors associated with diabetes status, a dependent variable with four categories. This model is a modification of the binary logistic regression recommended for modelling relationships of multinomial responses without imposing any restrictions on the ordinality of the response ${ }^{(27)}$. In interpreting the results calculated as OR, non-diabetic is the reference category $(\mathrm{OR}=1.00)$ to compare the estimates of the prediabetic, undiagnosed and diagnosed diabetic groups. For all analyses, $P<0.05$ was used to indicate statistical significance. Stata $14^{\circledR}$ (StataCorp) was used for the statistical analysis.

\section{Results}

Mean age was 67 years in the English sample and 62 years in the Brazilian sample. The characteristics of the samples are presented in Table 1. Nearly half of the English sample was prediabetic, and $9.6 \%$ had a diagnosis of diabetes, whereas the prevalence of UDD was low (3\%). The standardised prevalence showed that a total of $33 \%$ of the Brazilian sample had PD, and $20.0 \%$ had DD, whereas $6 \%$ (twice the proportion as that in the English sample) had UDD (Table 2).

In both samples, diagnosed diabetics were older than non-diabetics and prediabetics. English undiagnosed diabetics predominantly had less schooling and, along with diagnosed diabetics, had a lower frequency of daily drinkers, were more physically inactive, had higher frequencies of hypertension, stroke and obesity, a larger waist circumference, lower levels of HDL and higher levels of TAG than non-diabetics and prediabetics. Moreover, diagnosed diabetics had a greater frequency of hypertension and higher HbA1c levels than undiagnosed diabetics. 
Table 1 Characteristics of participants of English Longitudinal Study of Ageing (ELSA, 2012-2013) and Brazilian Longitudinal Study of Aging (ELSI, 2015-2016)

\begin{tabular}{|c|c|c|}
\hline & $\begin{array}{c}\text { ELSA } \\
(n 5301)\end{array}$ & $\begin{array}{c}\text { ELSI } \\
(n \text { 1947) }\end{array}$ \\
\hline \multicolumn{3}{|l|}{ Socio-economic variables } \\
\hline \multicolumn{3}{|l|}{ Age in years } \\
\hline Mean & $66 \cdot 7$ & $62 \cdot 2$ \\
\hline SD & 8.9 & 9.5 \\
\hline Female sex & $55 \cdot 1$ & $53 \cdot 2$ \\
\hline Skin colour (non-white) & 2.5 & $56 \cdot 4$ \\
\hline \multicolumn{3}{|l|}{ Schooling } \\
\hline $0-11$ years & $38 \cdot 1$ & $90 \cdot 0$ \\
\hline $12-13$ years & $28 \cdot 8$ & $2 \cdot 4$ \\
\hline 14 years or more & $33 \cdot 1$ & $7 \cdot 3$ \\
\hline Did not answer & - & 0.3 \\
\hline $\begin{array}{l}\text { Marital status (single, divorced or } \\
\text { widowed) }\end{array}$ & $32 \cdot 7$ & $34 \cdot 2$ \\
\hline \multicolumn{3}{|l|}{ Income in quintiles (\%) } \\
\hline 1st quintile & 23.5 & $26 \cdot 4$ \\
\hline 2nd quintile & 22.4 & $18 \cdot 2$ \\
\hline 3rd quintile & $21 \cdot 3$ & $18 \cdot 5$ \\
\hline 4th quintile & $18 \cdot 6$ & $20 \cdot 1$ \\
\hline 5th quintile & $14 \cdot 2$ & $16 \cdot 8$ \\
\hline \multicolumn{3}{|l|}{ Behavioural variables } \\
\hline \multicolumn{3}{|l|}{ Alcohol intake } \\
\hline Non-drinker & $18 \cdot 8$ & 91.1 \\
\hline Frequent drinker & 39.9 & 6.5 \\
\hline Daily drinkers & $33 \cdot 6$ & 2.4 \\
\hline Did not answer & $7 \cdot 7$ & \\
\hline \multicolumn{3}{|l|}{ Smoking } \\
\hline Non-smoker & 38.5 & $45 \cdot 8$ \\
\hline Ex-smoker & $50 \cdot 3$ & 38.9 \\
\hline Smokers & 11.2 & $15 \cdot 3$ \\
\hline Physically inactive & 3.5 & $28 \cdot 6$ \\
\hline \multicolumn{3}{|l|}{ Health conditions } \\
\hline Hypertension (yes) & 37.5 & 53.4 \\
\hline Stroke (yes) & 3.5 & 4.9 \\
\hline CVD (yes) & $15 \cdot 8$ & $13 \cdot 7$ \\
\hline \multicolumn{3}{|l|}{ Biochemical measures } \\
\hline \multicolumn{3}{|l|}{ Glycated Hb } \\
\hline Mean & 5.9 & $6 \cdot 1$ \\
\hline $\mathrm{SD}$ & 0.7 & 1.3 \\
\hline $\begin{array}{l}\mathrm{HDL}(<1.03 \mathrm{mmol} / / \mathrm{men} ;<1.29 \mathrm{mmol} / \mathrm{l} \\
\text { women) }\end{array}$ & $10 \cdot 5$ & $48 \cdot 1$ \\
\hline TAG $(\geq 1.69 \mathrm{mmol} / \mathrm{l})$ & $30 \cdot 1$ & $49 \cdot 3$ \\
\hline \multicolumn{3}{|l|}{ Anthropometrics } \\
\hline \multicolumn{3}{|l|}{$\mathrm{BMI}$} \\
\hline$<18.5 \mathrm{~kg} / \mathrm{m}^{2}$ & 0.8 & $2 \cdot 2$ \\
\hline$\geq 18.5$ and $<25 \mathrm{~kg} / \mathrm{m}^{2}$ & 27.4 & $27 \cdot 2$ \\
\hline$\geq 25$ and $<30 \mathrm{~kg} / \mathrm{m}^{2}$ & $42 \cdot 5$ & 37.8 \\
\hline$\geq 30 \mathrm{~kg} / \mathrm{m}^{2}$ & $29 \cdot 3$ & $32 \cdot 8$ \\
\hline $\begin{array}{l}\text { Waist circumference (>102 cm men; } \\
>88 \mathrm{~cm} \text { women) }\end{array}$ & $50 \cdot 4$ & $47 \cdot 9$ \\
\hline
\end{tabular}

All data presented as percentages, except where indicated: mean, SD. Self-reported doctor diagnosis of hypertension, stroke and CVD (angina, heart attack, congestive heart failure, vesicular murmur and arrhythmia).

Brazilian prediabetics had higher levels of HbA1c and a greater frequency of high TAG levels than non-diabetics. Undiagnosed diabetics had greater frequencies of obesity and abdominal obesity than non-diabetics and prediabetics. Undiagnosed diabetics and diagnosed diabetics had higher HbA1c and a lower frequency of arterial hypertension compared with non-diabetics and prediabetics. Moreover, diagnosed diabetics had higher levels of TAG than non-diabetics (see online supplementary material, Supplementary Tables 1 and 2).
Table 2 Prevalence of diabetes in ELSA study (2012-2013) and ELSI study (2015-2016)

\begin{tabular}{|c|c|c|c|c|}
\hline \multirow[b]{2}{*}{ Diabetes status } & \multicolumn{2}{|c|}{ ELSA } & \multicolumn{2}{|c|}{$\mathrm{ELSI}^{*}$} \\
\hline & $\%$ & $95 \% \mathrm{Cl}$ & $\%$ & $95 \% \mathrm{Cl}$ \\
\hline Non-diabetics & 38.8 & $37 \cdot 5-40 \cdot 1$ & $41 \cdot 0$ & $39 \cdot 3-43 \cdot 6$ \\
\hline Prediabetics & $48 \cdot 6$ & $47 \cdot 3-50 \cdot 0$ & 33.0 & $32 \cdot 8-36 \cdot 5$ \\
\hline Undiagnosed diabetic & $3 \cdot 0$ & $2 \cdot 5-3.5$ & $6 \cdot 0$ & $3.6-11.5$ \\
\hline Diagnosed diabetic & 9.6 & $8 \cdot 8-10 \cdot 4$ & $20 \cdot 0$ & $17 \cdot 3-24 \cdot 3$ \\
\hline
\end{tabular}

The prevalence was calculated with $95 \% \mathrm{Cl}$. ELSA study is the population standard. ${ }^{*}$ Standardised prevalence in ELSI study adjusted by age, sex and schooling.

In comparison between the included and excluded individuals of the analytical sample, those excluded from ELSI were older. Those excluded from ELSA were older, more frequently non-white, had lower schooling and income, less frequently had a conjugal life, consumed less alcohol and were more physically inactive, had higher HbA1c levels and a higher prevalence of hypertension, stroke and CVD, higher TAG levels, lower HDL levels, a higher BMI and waist circumference than the included individuals (see online supplementary material, Supplementary Table 3).

Table 3 displays the results of the multinomial regression models for factors associated with diabetes status in the English and Brazilian samples. The increase in age, non-white skin colour, smoking, obesity (BMI $\geq 30 \mathrm{~kg} / \mathrm{m}^{2}$ ) and abdominal obesity were associated with PD, UDD and DD in the English samples, with stronger associations found in those with UDD. These associations differed from those found in the Brazilian sample, in which the increase in age was associated with UDD and DD, nonwhite skin colour and smoking were associated only with UDD and abdominal obesity and hypertriglyceridaemia were associated with the three diabetic conditions.

Hypertriglyceridaemia, low HDL levels, hypertension and stroke were associated with both UDD and DD in the English sample. CVD in the English sample and low schooling in the Brazilian sample were associated with $\mathrm{PD}$ and DD. BMI $<18.5 \mathrm{~kg} / \mathrm{m}^{2}$ was associated with PD and lower income was associated with DD in the English sample. Being physically inactive was associated with DD in both samples. The female sex had a protective role regarding DD only in the English sample.

\section{Discussion}

The main findings of the current study were the differences in prevalence and associated factors between the two countries, with the rates of PD, UDD and DD, respectively, 48.6, 3 and 9.6\% in England and 33, 6 and $20 \%$ in Brazil. The common associated factors in the two samples were abdominal obesity and hypertriglyceridaemia among the prediabetics, undiagnosed diabetics and diagnosed diabetics; the increase in age among the undiagnosed diabetics and diagnosed diabetics; non-white skin colour 
Different prevalence of diabetes among countries

Table 3 Multinomial regression model according to prediabetes, undiagnosed diabetes and diagnosed diabetes individuals aged 50 years or older: ELSA study, 2012-2013 and ELSI study, 2015-2016

\begin{tabular}{|c|c|c|c|c|c|c|}
\hline & \multicolumn{2}{|c|}{ Prediabetes } & \multicolumn{2}{|c|}{ Undiagnosed diabetes } & \multicolumn{2}{|c|}{ Diagnosed diabetes } \\
\hline & \multicolumn{2}{|c|}{ OR (95 \% Cl) Adjusted } & \multicolumn{2}{|c|}{ OR (95 \% Cl) Adjusted } & \multicolumn{2}{|c|}{ OR (95 \% Cl) Adjusted } \\
\hline \multicolumn{7}{|l|}{ Age } \\
\hline ELSA & 1.05 & $1.04,1.06$ & 1.08 & $1.06,1 \cdot 10$ & 1.06 & $1.05,1.07$ \\
\hline ELSI & 1.02 & $0.99,1.04$ & 1.03 & $1.01,1.06$ & 1.04 & $1.02,1.07$ \\
\hline \multicolumn{7}{|l|}{ Female sex ( $v$. male) } \\
\hline ELSA & 1.08 & $0.95,1.23$ & 0.94 & $0.66,1.34$ & 0.65 & $0.52,0.82$ \\
\hline ELSI & 1.19 & $0.73,1.94$ & 0.70 & $0.35,1.42$ & 0.92 & $0.53,1.60$ \\
\hline \multicolumn{7}{|c|}{ Non-white skin colour ( $v$. white) } \\
\hline ELSA & 1.96 & $1.27,3.03$ & 9.77 & $4.85,19 \cdot 70$ & 3.63 & $1.98,6.65$ \\
\hline ELSI & $1 \cdot 16$ & $0.75,1.79$ & 2.55 & $1 \cdot 20,5.44$ & 1.09 & $0.79,1.52$ \\
\hline \multicolumn{7}{|l|}{ Income - ELSA } \\
\hline 1st quintile & 1.00 & & 1.00 & & 1.00 & \\
\hline 2nd quintile & $1 \cdot 15$ & $0.97,1.37$ & 0.83 & $0.47,1.47$ & 1.48 & $1 \cdot 04,2 \cdot 11$ \\
\hline 3rd quintile & 0.96 & $0.81,1.15$ & 1.04 & $0.61,1.76$ & 1.45 & $1.02,2.06$ \\
\hline 4th quintile & 1.04 & $0.86,1.25$ & 1.12 & $0.66,1.93$ & 1.55 & $1.08,2.22$ \\
\hline 5th quintile & 0.88 & $0.71,1 \cdot 10$ & 1.01 & $0.57,1.80$ & 1.88 & $1 \cdot 30,2 \cdot 73$ \\
\hline \multicolumn{7}{|c|}{ Schooling ( $v .14$ years or more) - ELSI } \\
\hline $12-13$ years & 7.72 & $2 \cdot 02,29.57$ & & & $7 \cdot 20$ & $1.65,31.39$ \\
\hline $0-11$ years & 1.33 & $0.77,2 \cdot 31$ & 2.47 & $0.73,8.34$ & 2.33 & $1.04,5 \cdot 23$ \\
\hline \multicolumn{7}{|c|}{ Physically inactive ( $v$. active) } \\
\hline ELSA & 0.88 & $0.60,1.29$ & 1.33 & $0.64,2.74$ & 1.75 & $1 \cdot 10,2 \cdot 80$ \\
\hline ELSI & 1.45 & $0.92,2.29$ & 1.38 & $0.76,2.50$ & 1.87 & $1 \cdot 23,2 \cdot 83$ \\
\hline \multicolumn{7}{|c|}{ Smoking ( $v$. non-smokers) } \\
\hline \multicolumn{7}{|c|}{ Ex-smokers } \\
\hline ELSA & 1.09 & $0.96,1.24$ & $1 \cdot 10$ & $0.75,1.61$ & 1.14 & $0.90,1.44$ \\
\hline ELSI & 0.97 & $0.62,1.52$ & 1.78 & $0.96,3.41$ & 1.61 & $0.87,2.98$ \\
\hline \multicolumn{7}{|l|}{ Smokers } \\
\hline ELSA & 2.40 & $1.92,2.99$ & $3 \cdot 12$ & $1.77,5.49$ & 1.88 & $1 \cdot 27,2 \cdot 77$ \\
\hline ELSI & 1.69 & $0.79,3.61$ & 3.36 & $1.02,5.43$ & 1.53 & $0.63,3.71$ \\
\hline \multicolumn{7}{|l|}{ Hypertension ( $v$. no) } \\
\hline ELSA & 1.04 & $0.91,1.19$ & $1 \cdot 76$ & $1 \cdot 23,2 \cdot 50$ & $2 \cdot 78$ & $2 \cdot 22,3.49$ \\
\hline \multicolumn{7}{|l|}{ Stroke ( $v$. no) } \\
\hline ELSA & $1 \cdot 28$ & $0.87,1.88$ & 2.05 & $1.01,4.19$ & 1.66 & $1.01,2.73$ \\
\hline \multicolumn{7}{|l|}{ CVD (v. no) } \\
\hline ELSA & $1 \cdot 20$ & $1.01,1.44$ & $1 \cdot 17$ & $0.75,1.81$ & 1.65 & $1 \cdot 26,2 \cdot 15$ \\
\hline \multicolumn{7}{|c|}{ High TAG $(v,<1.69 \mathrm{mmol} / \mathrm{l})$} \\
\hline ELSA & $1 \cdot 14$ & $0.99,1.32$ & 1.96 & $1.36,2 \cdot 81$ & 1.84 & $1.46,2.32$ \\
\hline ELSI & 2.08 & $1.39,3 \cdot 11$ & 3.27 & $1.84,5.81$ & 2.29 & $1.60,3.27$ \\
\hline \multicolumn{7}{|c|}{ Low HDL ( $v . \geq 1.03 \mathrm{mmol} / \mathrm{l} \mathrm{men;} \geq 1.29 \mathrm{mmol} / \mathrm{l}$ women) } \\
\hline ELSA & $1 \cdot 13$ & $0.90,1.43$ & $2 \cdot 20$ & $1.42,3.42$ & $2 \cdot 36$ & $1 \cdot 75,3 \cdot 18$ \\
\hline \multicolumn{7}{|c|}{$\mathrm{BMl}$ in $\mathrm{kg} / \mathrm{m}^{2}(v$, ideal range) ELSA } \\
\hline$<18.5 \mathrm{~kg} / \mathrm{m}^{2}$ & $2 \cdot 45$ & $1.22,4.91$ & & & 1.96 & $0.41,9.25$ \\
\hline$\geq 25$ and $<30 \mathrm{~kg} / \mathrm{m}^{2}$ & $1 \cdot 12$ & $0.95,1.31$ & 1.06 & $0.56,2.01$ & 0.99 & $0.69,1.41$ \\
\hline$\geq 30 \mathrm{~kg} / \mathrm{m}^{2}$ & 1.31 & $1.04,1.64$ & 2.52 & $1 \cdot 24,5 \cdot 13$ & $1 \cdot 81$ & $1 \cdot 19,2 \cdot 75$ \\
\hline \multicolumn{7}{|c|}{ Large waist circumference ( $v . \leq 102 \mathrm{~cm}$ men; $\leq 88 \mathrm{~cm}$ women) } \\
\hline ELSA & 1.53 & $1.30,1.81$ & $2 \cdot 82$ & $1.62,4.93$ & 2.40 & $1 \cdot 74,3 \cdot 30$ \\
\hline ELSI & 1.74 & $1 \cdot 16,2 \cdot 61$ & 5.36 & $2 \cdot 70,10 \cdot 65$ & 2.35 & $1 \cdot 44,3 \cdot 81$ \\
\hline
\end{tabular}

Dash (-) denotes the absence of category in group. ELSA model adjusted by sex, age, income, skin colour, level of physical activity and smoking. ELSI model adjusted by sex, age, schooling, skin colour, level of physical activity, smoking, TAG and waist circumference. In ELSI, Cl calculated considering sample weight.

and smoking among the undiagnosed diabetics and a sedentary lifestyle among the diagnosed diabetics.

The prevalence of diabetes was similar to rates described in epidemiological studies conducted in the two countries. Using data from the surveillance system of risk and protection factors for chronic diseases in Brazil, Mendes and collaborators ${ }^{(28)}$ found that the prevalence of diabetes among individuals older than 65 years of age was $18.6 \%$, which is a little higher than the rate found in the present study involving individuals aged 50 years or older. In a representative sample of 18399 adults in England with a mean age of 51 years, Moody and collaborators ${ }^{(29)}$ found that the prevalence of UDD and
DD was 2 and $6 \%$, respectively, which are similar to the rates found in the English sample of the present study.

The present results show several differences and some similarities regarding factors associated with diabetes status (prediabetes, undiagnosed and diagnosed), which may be explained by the process of nutritional transition, access to healthcare services, the use of such services and the consequent differences in mortality rates due to diabetes in the two populations studied. Countries undergo the nutritional transition process at different rates. This process is characterised by an increase in the consumption of SFA, trans fatty acids, sugar and sweetened beverages, along 
with a reduction in the consumption of complex carbohydrates, fruits, vegetables and legumes ${ }^{(30-33)}$. Moreover, changes in work structure and technological advances favour a sedentary lifestyle and the replacement of foods in natura for high-processed products, which contributes to obesity ${ }^{(30)}$. A sedentary lifestyle, smoking, obesity and metabolic disorders are key elements to the emergence of noncommunicable diseases, and all these factors were associated with diabetes status in both countries. Similar findings are described in previous studies ${ }^{(11,14,28,34)}$.

The greater number of individuals with the diabetes (diagnosed or undiagnosed) in Brazil may be the product of the rapid, intense nutritional transition occurring in developing countries, in which lower income and education levels lead to the greater consumption of high-energy foods ${ }^{(30)}$. Moreover, the difficulty on the part of the Brazilian healthcare system (particularly primary care) in dealing with the double burden of diseases in the population exerts a direct effect on the inadequate establishment of disease prevention and health promotion measures for type 2 diabetes, hindering early diagnosis and efficient treatment ${ }^{(35,36)}$.

In contrast, although the nutritional transition in England is mainly the result of dietary practices guided by practicality and convenience, characterised by the consumption of highly processed foods ${ }^{(32)}$, the country has a more effective healthcare system $^{(9)}$ and certainly performs more timely screening of older adults ${ }^{(37)}$. The threshold condition in the English sample may be explained by better health care that avoids to progression to diabetes per se.

Although both England and Brazil have universal healthcare systems with accessible medical care and strong primary care not found in other countries, expenditures on health per capita in Brazil correspond to only one-fourth of those in England, the density of physicians is much smaller and Brazilians have a poorer perception of their health in comparison to the English population ${ }^{(9)}$.

Diabetes often occurs concomitantly with lipid disorders, hypertension ${ }^{(38)}$, cardiovascular events ${ }^{(14,39)}$ and obesity $^{(38)}$, which is in agreement with the present findings. In the English sample, a poor lipid profile, hypertension and stroke were characteristics of individuals with diabetes (diagnosed or undiagnosed). Moreover, general and/or abdominal obesity was found in all three diabetes statuses. In Brazil, abdominal obesity and hypertriglyceridaemia occurred in all three diabetes statuses, with a stronger association with UDD.

Obesity can trigger insulin resistance and both conditions exert a negative impact on the metabolism of lipids, favouring the occurrence of CVD. Moreover, ethnic-racial differences may explain the occurrence of outcomes such as hypertension, heart disease and stroke in the English sample, the majority of which was white, compared with the Brazilian sample, in which such associations were not found, given the fact that blacks have a less atherogenic lipid profile ${ }^{(40)}$.
In agreement with the present findings, studies conducted in England have demonstrated the increase in age, non-white skin colour and low income to be associated with diabetes ${ }^{(29)}$. Brazilian studies also list these same factors $^{(28,41)}$. However, the lack of knowledge regarding diabetes control measures and reasons for not seeking healthcare services among Brazilian seniors has been associated with low schooling and income, resulting in poor disease control, with a significant impact on the occurrence of complications ${ }^{(28)}$. These issues may contribute to the high mortality rate due to diabetes in Brazil, which is twice the rate as that found in the English population ${ }^{(9)}$. Moreover, there is evidence that diabetes is underreported as a cause of death in Brazil, since people with diabetics die due to chronic complications of these diseases, which are listed as the main cause of death ${ }^{(28)}$. Therefore, one may put forth the hypothesis that, together with lower schooling and non-white skin colour, individuals who have little or no access to healthcare services may be unable to control diabetes and therefore die prematurely, resulting in such associations being found less in Brazil.

The present findings are particularly important, as PD is a high-risk state for the development of diabetes mellitus and because UDD increases the risk of complications due to the non-control of blood glucose levels. Thus, the three diabetes statuses pose risks of serious complications to the health of individuals aged 50 years or older. It is therefore essential to implement health policies and perform screening to minimise or avoid the harm caused by this condition.

The strong points of the current study are the use of large national representative samples of individuals aged 50 years or older and the use of HbA1c to confirm the cases. The current study also has limitations that should be considered. First, the cross-sectional design does not enable establishing causality because it not possible to establish the temporal sequence between outcome and exposure. Second, the lack of information on the time since the diagnosis of diabetes, and the characteristics of the excluded individuals such as worse socio-demographic, behavioural and health characteristics may have influenced the results. However, the findings agreed with the data reported in the literature. Finally, the use of self-reported clinical conditions could be a potential source of information bias. Perhaps due to limited contact with the medical system, individuals in different countries or across different socio-economic status groups may be unaware that they have a particular disease or may think they are cured when the disease is only under control. However, the participants were asked whether a doctor had diagnosed their condition, and this could have minimised inaccuracy and recall bias. Information on COmorbidities given by the patients has been described to be reliable especially for chronic conditions such as diabetes or heart disease ${ }^{(42)}$. Further studies should be conducted on the incidence of PD, UDD and DD, and their determinants. 


\section{Conclusion}

The prevalence of diabetes (diagnosed or undiagnosed) was higher in the Brazilian sample than the English sample. Different associated factors were found in the two samples, which may be related to differences in nutritional transition, access to healthcare services and the use of such services.

\section{Acknowledgements}

Acknowledgements: None. Financial support: This work was supported by the Fundação de Amparo á Pesquisa do Estado de São Paulo - FAPESP (The São Paulo Research Foundation) (2017/22820-0); Economic and Social Research Council (ESRC) and National Institute on Aging USA (5R01AG017644-16 and 5R01AG017644-18 to ELSA study); Brazilian Health Ministry and the Science and Technology Ministry (to ELSI study) and Conselho Nacional de Desenvolvimento Científico e Tecnológico CNPq (National Council of Scientific and Technological Development) (303981/2017-2 to Tiago da Silva Alexandre). Conflict of interest: The authors declare that there is no conflict of interest. Authorship: Study concept and design: T.S.A., E.S.M.d.S. and R.O.M. Acquisition of data: T.S.A., M.F.L.-C. and C.d.O. Data analysis and interpretation: T.S.A., E.S.M.d.S., R.O.M., M.F.L.-C. and C.d.O. Preparation of manuscript: All authors. Revising and final approval: All authors. Ethics of human subject participation: The current study was conducted according to the guidelines laid down in the Declaration of Helsinki and all procedures involving research study participants were approved by the The National Research Ethics Service (London Multicentre Research Ethics Committee (MREC/01/2/91) to ELSA study, and Human Research Ethics Committee of the René Rachou Research Centre of the Oswaldo Cruz Foundation (state of Minas Gerais, Brazil) (certificate number: 886.754) to ELSI study. Written informed consent was obtained from all subjects/patients.

\section{Supplementary material}

For supplementary material accompanying this paper visit https://doi.org/10.1017/S1368980020003201

\section{References}

1. International Diabetes Federation (2015) International Diabetes Federation. IDF Diabetes Atlas, 7th edn. Brussels, Belgium: International Diabetes Federation. doi: 10.1289/ image.ehp.v119.i03.

2. International Diabetes Federation (2013) Diabetes Atlas, 6th ed. Available from: https://www.idf.org/e-library/ epidemiology-research/diabetes-atlas/19-atlas-6th-edition.html (accessed July 2020).
3. Wild S, Roglic G, Green A et al. (2013) Definition and diagnosis of diabetes mellitus and intermediate hyperglycemia: report of a WHO/IDF consultation. BMC Public Health. doi: 10.1186/1471-2458-13-387.

4. Sue Kirkman M, Briscoe VJ, Clark N et al. (2012) Diabetes in older adults: a consensus report. J Am Geriatr Soc. doi: 10.1111/jgs.12035.

5. Chau D \& Edelman SV (2001) Clinical management of diabetes in the elderly. Clin Diabetes. doi: 10.2337/diaclin. 19.4.172.

6. American Diabetes Association (2010) Diagnosis and classification of diabetes mellitus. Diabetes Care 33, S62-S69.

7. Haffner SM (1990) Cardiovascular risk factors in confirmed prediabetic individuals. Does the clock for coronary heart disease start ticking before the onset of clinical diabetes? J Am Med Assoc 263, 2893-2898.

8. American Diabetes Association (2011) Diagnosis and classification of diabetes mellitus. Diabetes Care 34, S62-S69.

9. Lima-Costa MF, De Oliveira C, MacInko J et al. (2012) Socioeconomic inequalities in health in older adults in Brazil and England. Am J Public Health 102, 1535-1541.

10. Fisher-Hoch SP, Vatcheva KP, Rahbar MH et al. (2015) Undiagnosed diabetes and pre-diabetes in health disparities. PLoS One 10, 1-10.

11. Leahy S, O' Halloran AM, O' Leary N et al. (2015) Prevalence and correlates of diagnosed and undiagnosed type 2 diabetes mellitus and pre-diabetes in older adults: findings from the Irish Longitudinal Study on Ageing (TILDA). Diabetes Res Clin Pract 110, 241-249.

12. Xu Y, Wang LL, He J et al. (2013) Prevalence and control of diabetes in Chinese adults. JAMA 310, 948.

13. Najafipour H, Sanjari M, Shokoohi M et al. (2015) Epidemiology of diabetes mellitus, pre-diabetes, undiagnosed and uncontrolled diabetes and its predictors in general population aged 15 to 75 years: a community-based study (KERCADRS) in southeastern Iran. J Diabetes 7, 613-621.

14. Kumar A, Wong R, Ottenbacher KJ et al. (2016) Prediabetes, undiagnosed diabetes, and diabetes among Mexican adults: findings from the Mexican health and aging study. Ann Epidemiol 26, 163-170.

15. Mindell J, Biddulph JP, Hirani V et al. (2012) Cohort profile: the health survey for England. Int J Epidemiol 41, 1585-1593.

16. Steptoe A, Breeze E, Banks J et al. (2013) Cohort profile: the English longitudinal study of ageing. Int J Epidemiol $\mathbf{4 2}$, $1640-1648$.

17. Lima-Costa MF (2018) Aging and public health: the Brazilian Longitudinal Study of Aging (ELSI-Brazil). Rev Saude Publica 52, 1-3.

18. Von Elm E, Altman DG, Egger M et al. (2009) The strengthening the reporting of observational studies in epidemiology (STROBE) statement: guidelines for reporting observational studies. UroToday Int J 2, 806.

19. American Diabetes Association (2012) Diagnosis and classification of diabetes mellitus. Diabetes Care 35, S64-S71.

20. American Diabetes Association (2015) Classification and diagnosis of diabetes. Diabetes Care 38, S8-S16.

21. Bellou V, Belbasis L, Tzoulaki I et al. (2018) Risk factors for type 2 diabetes mellitus: an exposure-wide umbrella review of meta-analyses. PLoS One 13, e0194127.

22. Scholes S, Coombs N, Pedisic Z et al. (2014) Age- and sexspecific criterion validity of the health survey for England physical activity and sedentary behavior assessment questionnaire as compared with accelerometry. Am J Epidemiol 179, 1493-1502.

23. Banks J, Breeze E, Lessof C et al. (2006) Retirement, Health and Relationships of the Older Population in England: The 2004 English Longitudinal Study of Ageing (Wave 2). London Institute for Fiscal Studies. Available from: https:// discovery.ucl.ac.uk/id/eprint/15351/1/15351.pdf (accessed July 2020). 
24. National Institutes of Health (2000) The practical guide identification, evaluation, and treatment of overweight and obesity in adults. NHLBI Obes Educ Initiat. NIH Publication No. 00-4084.

25. Grundy SM, Becker D, Clark LT et al. (2002) Detection, evaluation, and treatment of high blood cholesterol in adults (Adult Treatment Panel III). Circulation. doi: 10.1161/circ.106.25.3143.

26. Naing NN (2000) Easy way to learn standardization: direct and indirect methods. Malays J Med Sci 7, 10-15.

27. Ananth CV \& Kleinbaum DG (1997) Regression models for ordinal responses: a review of methods and applications. Int J Epidemiol 26, 1323-1333.

28. Mendes TDAB, Goldbaum M, Segri NJ et al. (2011) Diabetes mellitus: factors associated with prevalence in the elderly, control measures and practices, and health services utilization in São Paulo, Brazil. Cad Saude Publica 27, 1233-1243.

29. Moody A, Cowley G, Fat LN et al. (2016) Social inequalities in prevalence of diagnosed and undiagnosed diabetes and impaired glucose regulation in participants in the Health Surveys for England series. BMJ Open. doi: 10.1136/ bmjopen-2015-010155.

30. Sartorelli DS \& Franco LJ (2003) Trends in diabetes mellitus in Brazil: the role of the nutritional transition. Cad Saude Publica. doi: 10.1590/s0102-311x2003000700004.

31. Amuna P \& Zotor FB (2008) Epidemiological and nutrition transition in developing countries: impact on human health and development. Proc Nutr Soc. doi: 10.1017/S0029665108006058.

32. Popkin BM \& Gordon-Larsen P (2004) The nutrition transition: worldwide obesity dynamics and their determinants. Int J Obes. doi: 10.1038/sj.ijo.0802804.

33. Batista Filho M \& Rissin A (2003) Nutritional transition in Brazil: geographic and temporal trends. Cad Saude Publica 19, S181-S191.
34. Brito IC, Lopes AA \& Araújo LMB (2001) Association of dark skin color with type 2 diabetes mellitus and the glucose intolerance in obese women of Salvador, Bahia. Arq Bras Endocrinol Metabol. doi: 10.1590/s0004-273020010005 00011.

35. Costa AF, Flor LS, Campos MR et al. (2017) Burden of type 2 diabetes mellitus in Brazil. Cad Saude Publica. doi: 10.1590/ 0102-311x00197915.

36. Lebrão ML (2007) Aging in Brazil: aspects of demographic and epidemiological transition. Saúde coletiva 4, 135-140.

37. Pierce MB, Zaninotto P, Steel $\mathrm{N}$ et al. Undiagnosed diabetes: data from the English longitudinal study of ageing. Diabet Med 26, 679-685.

38. Flor LS \& Campos MR (2017) The prevalence of diabetes mellitus and its associated factors in the Brazilian adult population: evidence from a population-based survey. Rev Bras Epidemiol. doi: 10.1590/1980-5497201700010002.

39. Pérez CM, Soto-Salgado M, Suárez E et al. (2015) High prevalence of diabetes and prediabetes and their coexistence with cardiovascular risk factors in a Hispanic community.JImmigr Minor Heal 17, 1002-1009.

40. Bentley AR \& Rotimi CN (2017) Interethnic differences in serum lipids and implications for cardiometabolic disease risk in African ancestry populations. Glob Heart. doi: 10.1016/ j.gheart.2017.01.011.

41. Ferreira CLRA \& Ferreira MG (2009) Epidemiological characteristics of diabetic patients within the public health system an analysis of the HiperDia system. Arq Bras Endocrinol Metabol. doi: 10.1590/s0004-27302009000100012.

42. Lucke T, Herrera R, Wacker M et al. (2016) Systematic analysis of self-reported comorbidities in large cohort studies: a novel stepwise approach by evaluation of medication. PLoS One 11, e0163408. 